\title{
Study of the Magnetic Water Treatment Mechanism
}

\author{
Iryna Vaskina1", Ihor Roi ${ }^{1}$, Leonid Plyatsuk', Roman Vaskin', Olena Yakhnenko \\ 1 Sumy State University, Sumy, Ukraine \\ * Corresponding author's e-mail: irulik.vaskina@gmail.com
}

\begin{abstract}
The main problem of widespread introduction of magnetic water treatment (MWT) in the processes of water and wastewater treatment is the lack of modern research aimed at studying the mechanisms of MWT effects, in particular the influence on the physicochemical properties of aqueous solutions. This study explains the effect of MWT taking into account the physical and chemical properties of aqueous solutions due to the presence of the quantum differences in water molecules. All of the MWT effects are related to the change in the physicochemical properties of aqueous solutions. It is due to the presence of two types of water molecule isomers and their librational oscillations. The result of MWT is a violation of the synchronism of para-isomers vibrations, with the subsequent destruction of ice-like structures due to the receiving of energy from collisions with other water molecules (ortho-isomers). One of the most important MWT effects includes the change in the nature and speed of the physicochemical processes in aqueous solutions by increasing the number of more physically and chemically active ortho-isomers. The MWT parameters specified in the work allow explaining the nature of most MWT effects and require developing the scientific and methodological principles for the implementation of the MWT process and mathematical modeling of the MWT process in the water and wastewater treatment. It can be used in the design of the MWT devices taking into account the constructive and mode parameters of MWT devices.
\end{abstract}

Keywords: wastewater, aqueous solutions, water isomers, magnetic induction, non-equilibrium system, librational fluctuations

\section{INTRODUCTION}

The studies on the constant magnetic field impact on the processes of aqueous solutions treatment have been ongoing for many years and remain relevant today. The current research is focused on reducing the formation of hard scales at elevated temperatures on heat transfer surfaces [Lipus, et al., 2013; Bali, et al., 2016; Chibowski, et al., 2018; Koshoridze, et al., 2014; Kozic, et al., 2010]. Much less attention is paid to other effects of the magnetic field on the physicochemical processes in aqueous solutions. Such processes are fundamental and can find wide practical application in the wastewater treatment [Zaidi, et al., 2013].

The main problem on the magnetic water treatment (MWT) implementation is the lack of up-to-date studies on the mechanisms of the MWT effects, in particular the appearance of changes in the physicochemical properties of aqueous solutions. Most known hypotheses relate the MWT mechanism to the polarizing effect of the magnetic field on water molecules and solutes (gases, impurities, organic matter, anions and cations). There are known data on the manifestation of the effects of MWT in the treatment of chemically pure aqueous solutions (distillate, bidistillate). The effects of MWT also occur in untreated aqueous solutions with part of an aqueous solution pre-treated with MWT [Lipus, et al., 2013]. Different interpretations of the mechanisms of MWT effects do not determine the general principles of the process, consider the effects of MWT independently and cannot simultaneously explain all known effects of MWT. The situation is further complicated by the low reproducibility of the MWT effects [Klassen, 1982].

Most of the MWT effects are observed in the treatment of aqueous solutions with a temperature 
below $343 \mathrm{~K}$. All known anomalies of water properties (density, heat capacity, thermal conductivity, etc.) are observed in such temperature range. The use of recent studies on the presence of quantum differences in water molecules and nature of the rotational oscillations of water molecules, which are responsible for the thermodynamic properties of aqueous solutions in the temperature range from 273 to $343 \mathrm{~K}$, will enable to understand the mechanism of MWT. More detailed physical model of the MWT mechanism allows us to form a generally accepted MWT mechanism to describe the nature of the appearance of MWT effects.

The effects of MWT have a complex dependence on the MWT parameters [Lipus, et al., 2007; Bali, et al., 2016; Chibowski, et al., 2018; Zaidi, et al., 2013; Klassen, 1982]. It does not increase in proportion with the increase in the intensity of the magnetic field (magnetic induction). The large amount of MWT data, their volatility and practical relevance [Koshoridze, et al., 2014; Lipus, et al., 2013; Kozic, et al., 2010; Sammer, et al., 2016; Liu, et al., 2015; Seyfi, et al., 2017; Al-Ogaidi, et al., 2017; Szcześ, et al., 2011; Rashid, et al., 2013] have led to different hypotheses of the MWT mechanism and can be roughly classified into three groups (see Table 1).

There is no conventional approach to the interpretation of the nature of the physical and chemical effects of MWT, no general principles of the process or the criteria for choosing the optimal design parameters of MWT devices. It led to a large variety of the MWT devices, which differ in a number of features: the type and shape of magnets, the type of water movement in a magnetic field, way of creating a magnetic field, completeness of using the magnetic field, scope, conditions of use and design features. Long-term operation of MWT devices has proven that irrespective of the MWT devices type, the motion of an aqueous solution in a non-uniform magnetic field (the presence of sections with a magnetic induction drop) is on the base for their workflow.

Movement of the aqueous solution is a prerequisite for MWT process. The velocity of the aqueous solution is one of the determining parameters. With zero velocity of aqueous solution, for any rate of induction drop, the induction of the magnetic field remains constant for each unit of the water volume. From a physical point of view, it is clear that the velocity directly affects the magnitude of the magnetic induction drop per unit volume of the solution. The maximum magnitude of the magnetic field induction is not so important. It coincides with the conclusions reported in [Klassen, 1982; Sammer, et al., 2016; Plyatsuk, et al., 2019], where the effect of MWT was observed at low induction values. The geometry of the magnetic field and the inhomogeneity of the magnetic induction distribution along the pipe for the passage of the aqueous solution are one of the basic design parameters of MWT devices, which depend on the characteristics of the magnets and their location along the water trajectory. The MWT efficiency has also been influenced by the duration of treatment, the physical parameters of water (temperature), mineral and dispersed composition [Chibowski, et al., 2018; Klassen, 1982].

The effects of MWT include the changes in the kinetics of reactions, hydration of solutes, the rate of evaporation, changes in density, strength and surface tension of the aqueous solution [Lipus, et al., 2007; Lipus, et al., 2013; Bali, et al., 2016; Chibowski, et al., 2018; Koshoridze, et al., 2014; Kozic, et al., 2010; Zaidi, et al., 2013; Koksharov, et al., 1990; Bali, et al., 2018; Sammer, et al., 2016; Liu, et al., 2015; Toledo, et al., 2008; Seyfi, et al., 2017; Al-Ogaidi, et al., 2017; Szcześ, et al., 2011; Rashid, et al., 2013]. Such changes in the physicochemical properties of aqueous solutions are caused by the decrease in the energy of $\mathrm{H}$-bonds in the interactions of water molecules

Table 1. Classification of the hypotheses of the MWT mechanism

\begin{tabular}{|c|c|c|c|}
\hline $\begin{array}{c}\text { Group of } \\
\text { approaches }\end{array}$ & Colloidal & Ionic & Water \\
\hline Impact object & $\begin{array}{l}\text { Colloidal particles including } \\
\text { ferromagnetic }\end{array}$ & lons that are present in water & Water molecules \\
\hline Mechanisms & $\begin{array}{l}\text { The surface properties of such } \\
\text { particles changes and they act } \\
\text { as crystallization centers under } \\
\text { the magnetic field influence. This } \\
\text { group explains the anti-scale } \\
\text { effect. }\end{array}$ & $\begin{array}{l}\text { Under the influence of a } \\
\text { magnetic field, polarization } \\
\text { and defor-mation of ions occur, } \\
\text { which are accompanied by their } \\
\text { hydration decrease, which affects } \\
\text { the course of physicochemical } \\
\text { processes in aqueous systems. }\end{array}$ & $\begin{array}{l}\text { This group combines ideas } \\
\text { about the possible influence of } \\
\text { a magnetic field on the water } \\
\text { structure. This influence, on the } \\
\text { one hand, can cause changes in } \\
\text { the aggregation of water mole- } \\
\text { cules, and on the other hand, etc. }\end{array}$ \\
\hline
\end{tabular}


with each other and around solutes. However, the external energy supplied to the aqueous solution is not sufficient to directly break or weaken the $\mathrm{H}$-bonds in accordance to the value of magnetic induction during MWT. The changes caused by the inhomogeneous magnetic field can be associated with a decrease in H-bond energy, increasing their length and changes of the angle of the H-bond [Zaharov, et al., 2011]. The structure of the water molecules themselves, the distribution of their electron density and the nature of their interaction with each other and with solutes becomes important.

The heterogeneity of water density caused by the presence of the ice-like and densely packed structures in water was revealed by [Zaharov, et al., 2011; Voloshyn, et al., 2001]. The first type of such structures has the correct tetrahedral coordination of the adjacent molecules that form four $\mathrm{H}$ bonds. Unlike the ice-like structures, the tightly packed structures of water molecules with broken H-bonds are arranged more tightly together and connected with stronger $\mathrm{H}$-bonds. In the tightly packed structures, the water molecules form one donor and one acceptor H-bonds. Such structures have the appearance of branched chains, in which the mobility of the water molecules is much greater than in the structures with the correct tetrahedral environment. The duration of the existence of $\mathrm{H}$-bonds is significantly smaller.

The presence of ortho- and a para- spin isomers of water in different rotational states was established with different methods in [Horke, et al., 2014; Mamrashev, et al., 2018; Kravchuk, et al., 2011; Hama, et al., 2016; Pershin, et al., 2018; Kilaj, et al., 2018]. An aqueous solution is a complex system of molecular complexes with a variable configuration of structural bonds and monomers that differ in the quantum and physical properties. According to the Boltzmann distribution, it is more energy-efficient at the room temperature for para of isomers (has no magnetic moment) to form $4 \mathrm{H}$-bonds than for the ortho- isomers (has a magnetic moment), which always rotate and form only one donor and one acceptor H-bonds.

There are quantum differences of the ortho/para spin-isomers of water molecules on the basis of the physical properties of water [Pershin, 2012]. The values of the critical water temperatures $\left(4,19,36.6^{\circ} \mathrm{C}\right.$, etc.) satisfy the resonant condition of equality of the kinetic energy of the ortho/para spin-isomers of water and the energy of their rotating quantum. The ratio of ortho/para spin isomers in water is non-equilibrium $(1: 1)$, which indicates its unstable metastable state. It is a physical factor of high variability, abnormally large fluctuations and the ability to establish equilibrium ortho/para ratios (3: 1) and enrichment ortho-isomers of water (para-ortho conversion).

A model of interaction and dynamics of molecules in water in the framework of the theory of the Jan-Teller effect was proposed in [Malafaev, et al., 2013; Malafaev, et al., 2015; Malafaev, 2011]. It showed that the major contribution to the properties of water is made by the collective librational vibrations of the molecules that appear during the transition from the ice to the liquid state, which lead to the occurrence of hydrogen protons around the lines of intermolecular bonds (in elliptical trajectory (ET)) and accompanied by dynamic bending of $\mathrm{H}$-bonds. The libration vibrations of water molecules are large in amplitude, two-dimensional, bound and ordered. The presence of these fluctuations in water leads to high values of heat capacity, thermal conductivity, and thermal expansion anomalies. The rotation of local charges of the hydrogen protons around the lines of intermolecular bonds leads to the appearance of magnetic fields along the $\mathrm{H}$-axis. For the unidirectional rotation of protons of a single molecule, magnetic fields are formed along the axis of the molecule on which the oxygen atom is located and cause it to rotate. In the case of the directional rotation of the protons, the magnetic fields are superimposed and compensate each other. The total magnetic field of the molecule is zero. These magnetic fields can be fixed by electron paramagnetic resonance and nuclear magnetic resonance methods as a chemical shift.

The aim of this study was to physically substantiate the mechanism of the MWT effects taking into account the quantum differences of water molecules and the nature of $\mathrm{H}$-bonds distribution in supramolecular formations due to the presence of libration vibrations of water molecules.

The following tasks were set:

1. To conduct a systematic analysis of the recent studies on the properties of aqueous solutions and create a single model of an aqueous solution based on the quantum differences in water molecules, the presence of rotational vibrations of water molecules, as well as the cooperativity and synchrony of libration vibrations of water molecules in supramolecular.

2. To determine the causes and conditions of inhomogeneous magnetic field influence on 
the structure and physicochemical properties of aqueous solutions caused by the changes in the libration vibrations of water molecules and the appearance of the natural para-ortho conversion.

\section{RESULTS AND DISCUSSION}

Known considerations and hypotheses of different authors about the mechanism of permanent magnetic field influence on water and its impurities in a state of thermodynamic equilibrium are mainly based on the polarizing effect of the magnetic field on ions and water molecules. However, during the contact of water with a relatively weak magnetic field, some changes occurred in the aqueous solution, which become possible only in thermodynamically nonequilibrium systems.

The presence of two protons of hydrogen and the mutual orientation of their spins causes the appearance of different states of water molecules in aqueous solutions. The mutual orientation of the spins of water molecule protons leads to the existence of two independent ortho- and a para-states of water molecules. Such states are characterized by a long lifetime and cause the appearance of two types of H-bonds. The stability of their existence is conditioned by the existence of supramolecular formations [Kuyanov-Prozument, et al., 2010]. Both protons of some molecules rotate in one direction (ortho-isomer - ortho- $\mathrm{H}_{2} \mathrm{O}$ ), while others - in opposite directions (para-isomer - para- $\mathrm{H}_{2} \mathrm{O}$ ). Both states are characterized by virtually identical chemical properties, but differ slightly in their physical properties in the infrared and nuclear magnetic resonance spectra. Ortho$\mathrm{H}_{2} \mathrm{O}$, unlike para- $\mathrm{H}_{2} \mathrm{O}$, has momentum amounts of motion, are poorly adsorbed on some types of surfaces and are in a higher energy state. It indicates significant differences in their behavior and chemical interactions. The exact differences between them and the nature of their appearance remain poorly understood, but their relationship determines the physicochemical properties and structure of the aqueous solution.

The ortho or para states of water molecules can be caused by the presence of collective and non-attenuating libration vibrations of water molecules (Fig. 1). It has been identified recently and all the anomalies of the physicochemical properties of water in the temperature range up to $343 \mathrm{~K}$ were well described. The libration oscillations are accompanied by a dynamic bending of H-bonds and stable rotation of proton charges around the H-bond axes on ET. In this case, in some molecules, both protons rotate in the same direction, while in others, the rotation occurs in opposite directions. Such rotations result in the formation of magnetic fields along the H-bond axes, which add up or compensate for each other along the water molecule, in the same way as ortho- $\mathrm{H}_{2} \mathrm{O}$ and para- $\mathrm{H}_{2} \mathrm{O}$.

The rotation of protons in opposite directions on para- $\mathrm{H}_{2} \mathrm{O}$ during ice melting allows the water molecules to form four H-bonds and to save the ice-like structures with proper tetrahedral coordination of adjacent molecules. It is natural to expect that thermal motion and fluctuations in water destroy structural complexes, which are mainly formed by para- $\mathrm{H}_{2} \mathrm{O}$, and complexes with ice structures. At the same time, the ice structures are formed without defects due to synchronization in the frequency and direction of the vibrational vibrations of molecules in the 2 nd and $3 \mathrm{rd}$ coordination.

A gradual increase in the temperature of solutions accompanied by a decrease in the synchronization of the librational vibrations in the ice structures and their destruction. This results in the release of para- $\mathrm{H}_{2} \mathrm{O}$ and an increase in the amount of ortho- $\mathrm{H}_{2} \mathrm{O}$ (see Fig. 2).

The change in the ortho/para ratio is due to the involuntary process of para-ortho conversion. The process involves a change the direction of rotation of one of the protons in para- $\mathrm{H}_{2} \mathrm{O}$ when molecules collide. Conversion becomes possible under the condition of resonant exchange of thermal energy of molecules motion with the energy of the rotating quantum ortho- $\mathrm{H}_{2} \mathrm{O}$ or „free" para- $\mathrm{H}_{2} \mathrm{O}$ [Pershin, 2012]. Increasing the amount of ortho- $\mathrm{H}_{2} \mathrm{O}$ leads to the formation of the tightly packed structures that look like branched chains. In such structures, the water molecules are arranged tightly to each other and connected by stronger H-bonds. The mobility and chemical activity of such water molecules is much greater due to the presence of rotations and constant collisions with the neighboring molecules during thermal motion. The duration time of H-bonds is substantially shorter, which is consistent with [Zaharov, et al., 2011; Voloshin, et al., 2001].

The dissolved substances in water change the structural temperature of the aqueous solutions of pure water by the polarization of water molecules. This process affects the para-ortho conversion in 


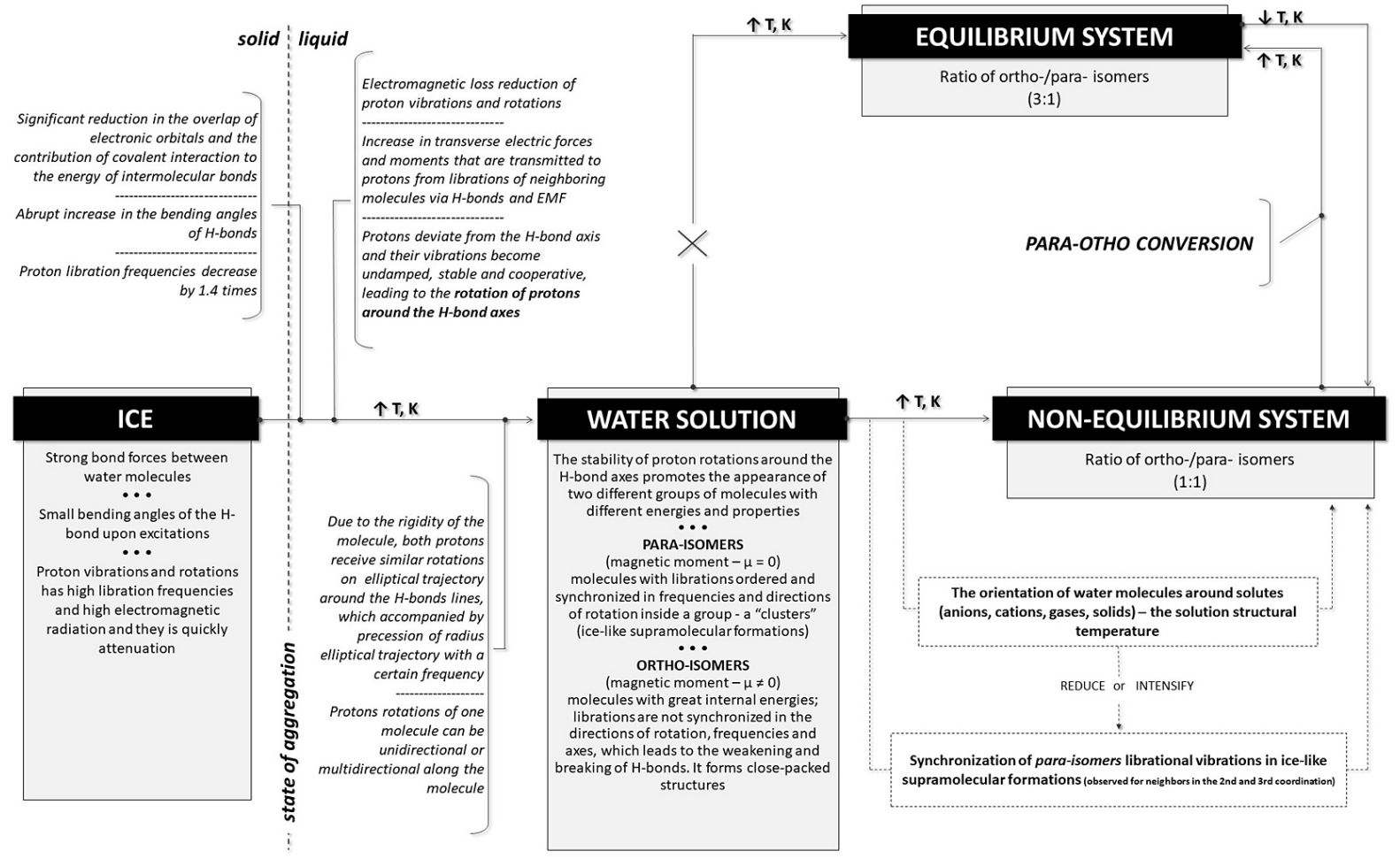

Figure 1. System analysis of recent studies of the aqueous solutions structural properties

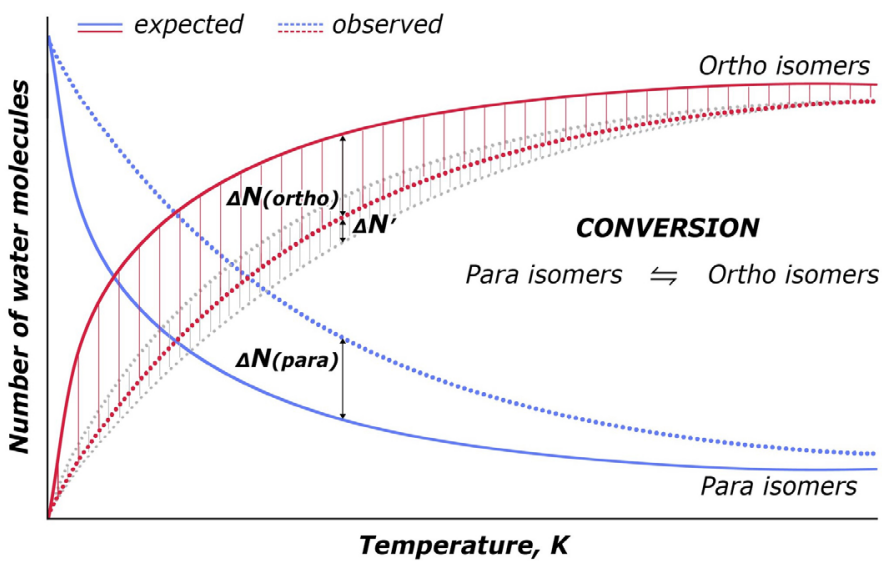

Figure 2. Schematic representation of setting of the equilibrium ortho/para ratio $\Delta \mathrm{N}$ - the shift in the number of ortho(para) isomersfrom the equilibrium ortho/para ratio;

$\Delta \mathrm{N}^{\prime}$ - additional number of ortho-isomers caused by polarization of water molecules around of dissolved substances

the near hydration region ( $\left.\Delta \mathrm{N}^{\prime}\right)$, Figure 2 . Hydration can be both positive and negative and causes two mutually opposite effects. On the one hand, by increasing the entropy of water molecules, the synchronization of the libration oscillations of para- $\mathrm{H}_{2} \mathrm{O}$ in ice structures is disturbed. It has a disruptive effect and enhances the para-ortho conversion. On the other hand, the action of the particles' magnetic field orientates the water molecules in this field in a certain way. Such process is accompanied by a decrease in entropy and an increase in the synchronization of the librational vibrations of para- $\mathrm{H}_{2} \mathrm{O}$ and the stability of the icelike structures. As the temperature rises, the contribution of the structural temperature decreases and the thermal motion of water molecules begins to play an increasing role.

According to the classical quantum theory, the aqueous solution, like any dynamic system, tends to equilibrium ortho/para ratio and it should be $3: 1$. Hydrogen consists of approximately $75 \%$ ortho-hydrogen and $25 \%$ para-hydrocarbon at the 
temperatures above $0^{\circ} \mathrm{C}$. At low temperatures, most molecules have only kinetic energy, and the rotational heat (part of the heat that is rotational motion) is zero, and the ratio between ortho$\mathrm{H}_{2} \mathrm{O}$ and para- $\mathrm{H}_{2} \mathrm{O}$ changes in favor to para- $\mathrm{H}_{2} \mathrm{O}$ [Meier, et al., 2018].

The stability of the ice structures due to the synchronization of cooperative libration oscillations of para- $\mathrm{H}_{2} \mathrm{O}$ and the redistribution of the energy from received collisions with other molecules, imposes a „ban” on resonant energy exchange during the collisions of molecules and slows the para-ortho conversion (Fig.2, dashed line). An aqueous solution that formed after melting ice, within temperatures from 272 to $343 \mathrm{~K}$, is a non-equilibrium dynamic system in which the ortho/para ratio is displaced to $1: 1$ toward para$\mathrm{H}_{2} \mathrm{O}$ [Pershin, 2012]. It is expected that such a system always seeks to establish an equilibrium state and is sensitive to the influence of external factors, including the presence of dissolved substances, the influence of physical fields, changes in temperature etc.

The influence of the inhomogeneous magnetic field on the aqueous solution, which is a nonequilibrium system in the ortho/para ratio, does not lead to a direct rupture of the H-bonds, as expected in the previous work. Taking into account the presence of constant non-attenuating rotations of the local charges of hydrogen protons along the ET line around the H-bond, the influence of the inhomogeneous magnetic field on the aqueous solution is connected with the appearance of an additional precession ET [Plyatsuk, et al., 2019]. Due to the arbitrary orientation of water molecules, precession is not the same for para- $\mathrm{H}_{2} \mathrm{O}$ in the ice structures, which causes asymmetry of the librational vibrations and impairs their synchronicity and cooperativity.

MWT is a «catalyst» for a series of sequential processes (see Table 2) - the violation of the librational vibrations of para- $\mathrm{H}_{2} \mathrm{O}$, the destruction of ice structures, the appearance of para-ortho conversion. The para-ortho conversion is made possible after MWT by removing the non-radiative quantum transition ban by reducing the difference between thermal motion energy and hydrogen proton rotation energy. The increase of ortho- $\mathrm{H}_{2} \mathrm{O}$ explains the changes in density, viscosity, infrared water spectrum, hydration of solutes, etc.

The absence of the MWT effects when using higher magnetic induction values [Klassen, 1982;
Lipus, et al., 2007; Roi, 2014] with constant other mode and design parameters of MWT, is explained by the presence of a magnetic moment of proton rotation over ET, which is an integral property of a water molecule. During the movement of the water molecule in the magnetic field, an external field is superimposed on the proton and the magnetic field of the proton rotation is «oriented» as a magnet in an external magnetic field. The energy of interaction of the magnetic moment of proton rotation by ET with an external magnetic field is defined as:

$$
W=\mu_{E T} B
$$

where: $\mu_{E T}$ - magnetic moment of hydrogen proton rotation over $\mathrm{ET}, \mathrm{J} / \mathrm{T}$;

$B$ - induction of an external magnetic field, $\mathrm{Tl}$.

The para-ortho conversion becomes possible with the collisions of isomers and resonant exchange of the energies of thermal motion and rotational quantum. The conversion is possible only if these energies are equal. This equality is disturbed as the interaction of the proton with the external magnetic field increases and the orientation of the magnetic moment of rotation of hydrogen protons rotation in the direction of the field is stabilized. Thus, during MWT for para- $\mathrm{H}_{2} \mathrm{O}$, in which the magnetic moments of rotation of hydrogen protons interact with the external field, the difference between the energies of the rotating quantum and the thermal motion increases. Thus, the number of effective collisions of the molecules leading to conversion decreases.

The contribution of MWT to the para-ortho conversion process decreases with the increasing temperature. The thermal motion of water molecules in the natural para-ortho conversion process begins to play an increasing role. On such background, the higher effect of MWT is observed at low solution temperatures and decreases if the temperature is increased.

The previous experimental studies [Roi, 2014] formed the basis of the proposed hypothesis and partially confirm the developed positions. Further refinement confirmed the dependence of the MWT efficiency on the physicochemical properties of the treated solution and a number of regime parameters of MWT. The experimental results are still being processed and being prepared for publication, so they are not given in this article. 
Table 2. Influence of MWT on aqueous solution

\begin{tabular}{|c|c|c|c|}
\hline $\begin{array}{l}\text { Factor of } \\
\text { influence }\end{array}$ & Object of influence & Results of influence & Description \\
\hline $\begin{array}{l}\text { Lorentz } \\
\text { force in } \\
\text { non-uniform } \\
\text { magnetic field } \\
\text { [Plyatsuk, et } \\
\text { al. 2019] }\end{array}$ & $\begin{array}{l}\text { Precession of the } \\
\text { elliptical trajectory } \\
\text { radius of the hydrogen } \\
\text { proton rotation around } \\
\text { the } \mathrm{H} \text {-bond line }\end{array}$ & $\begin{array}{l}\text { Violation of the } \\
\text { frequency of precession } \\
\text { of the elliptical trajectory } \\
\text { radius of the hydrogen } \\
\text { proton rotation for } \\
\text { neighboring water } \\
\text { molecules }\end{array}$ & $\begin{array}{l}\text { The nature of the precession frequency change } \\
\text { is related to the emergence of the Lorentz force } \\
\text { component, which becomes possible only in the } \\
\text { non-uniform magnetic field }(\Delta \mathrm{B} / \Delta \mathrm{t}) \text {. The change } \\
\text { in the magnitude of the magnetic induction is } \\
\text { accompanied by an increase or decrease in the } \\
\text { density of the magnetic induction lines in the } \\
\text { middle of the elliptical trajectory (ET) circuit, and } \\
\text { their «movement» perpendicular to the circuit } \\
\text { of the ET. The intersection of the ET circuit is } \\
\text { accompanied by the appearance of a Lorentz } \\
\text { force, which affects the proton of hydrogen at } \\
\text { each point on the ET and shifts the ET rotation } \\
\text { radius. The shift of the ET rotation radius and } \\
\text { consequently a change in the speed (frequency) } \\
\text { of its precession are different for neighboring } \\
\text { water molecules due to the different orientation } \\
\text { with respect to the magnetic induction lines, } \\
\text { which causes disturbance of the synchronization } \\
\text { and cooperativity of the libration vibrations of the } \\
\text { neighboring water molecules. }\end{array}$ \\
\hline \multirow{6}{*}{$\begin{array}{l}\text { Rate of } \\
\text { magnetic } \\
\text { induction } \\
\text { change } \\
(\Delta \mathrm{B} / \Delta \mathrm{t})\end{array}$} & $\begin{array}{l}\text { Non-equilibrium } \\
\text { system (aqueous } \\
\text { solutions in the } \\
\text { temperature range } \\
\text { from } 273 \text { to } 343 \mathrm{~K} \text { ) }\end{array}$ & $\begin{array}{l}\text { The emergence of } \\
\text { additional para/ortho } \\
\text { conversion }\end{array}$ & $\begin{array}{l}\text { MWT is a «catalyst» for the emergence } \\
\text { of sequential processes: the disruption of } \\
\text { synchronous oscillations of water molecules in } \\
\text { supramolecular formations, which by collision } \\
\text { with other molecules receive excess energy } \\
\text { and are destroyed, which leads to the release } \\
\text { of para-isomers of water molecules and their } \\
\text { subsequent conversion into ortho-isomers. }\end{array}$ \\
\hline & \multirow{3}{*}{$\begin{array}{l}\text { Para-isomers ice- } \\
\text { like supramolecular } \\
\text { formations }\end{array}$} & $\begin{array}{l}\text { Loss of synchrony of } \\
\text { oscillations with adjacent } \\
\text { molecules }\end{array}$ & \multirow{3}{*}{$\begin{array}{l}\text { The shift of the ET rotation radius and } \\
\text { consequently a change in the speed (frequency) } \\
\text { of its precession are different for neighboring } \\
\text { para-isomers in ice-like supramolecular } \\
\text { structures. The loss of cooperativity of the } \\
\text { oscillations of the para-isomers leads to the } \\
\text { destruction of the ice-like structures and release } \\
\text { of para-isomers, which in subsequent collisions } \\
\text { with other water molecules and the resonant } \\
\text { exchange of thermal motion energies and } \\
\text { rotating quantum transforms into ortho-isomers. }\end{array}$} \\
\hline & & $\begin{array}{l}\text { Destruction of ice- } \\
\text { like supramolecular } \\
\text { formations and release } \\
\text { of para-isomers }\end{array}$ & \\
\hline & & $\begin{array}{l}\text { The appearance of } \\
\text { additional para-ortho } \\
\text { conversion during } \\
\text { isomer collisions }\end{array}$ & \\
\hline & \multirow{2}{*}{$\begin{array}{l}\text { Ortho- or para-isomers } \\
\text { in hydration shells } \\
\text { around dissolved } \\
\text { substances that cause } \\
\text { polarization of water } \\
\text { molecules }\end{array}$} & $\begin{array}{l}\text { Loss of synchrony of } \\
\text { oscillations with adjacent } \\
\text { water molecules }\end{array}$ & \multirow{2}{*}{$\begin{array}{l}\text { Dissolved substances in aqueous solutions } \\
\text { (anions, cations, gases, solids, organics } \\
\text { molecules, etc.) cause displacement of the ortho/ } \\
\text { para ratio, attenuation or enhancement of the } \\
\text { libration vibrations of the water molecules, which } \\
\text { may affect the MWT efficiency. }\end{array}$} \\
\hline & & $\begin{array}{l}\text { Changes in the dissolved } \\
\text { substances hydration }\end{array}$ & \\
\hline
\end{tabular}

The effect of long-term conservation of any changes in aqueous solutions under the action of relatively weak magnetic fields («magnetic memory») is connected with the increase of ortho- $\mathrm{H}_{2} \mathrm{O}$ after MWT and the quantum-mechanical ban on the spontaneous conversion of spin-isomers of water molecules that becomes impossible under normal conditions. However, in practice, all changes in the physicochemical properties of aqueous solutions after MWT persist from several hours to several days [Klassen, 1982; Lipus, et al., 2007, Colic, et al., 1999] with a gradual return to their original state. The latter indicates the emergence of an ortho/para convertion, which, despite the ban, takes place in the presence of catalysts with a magnetic moment (atoms, ions, molecules, etc.) [Pershin, 2012], and decelerates markedly in their absence.

On the one hand, the $\mathrm{H}$-bonds between water molecules in experimental solutions (conditionally «pure») are equivalent to the influence of an external inhomogeneous magnetic field. On the other hand, industrial wastewater is a multicomponent solution and always contains impurities of different nature. Solid impurities, gas molecules, minerals and organic matter cause 
different polarization of water molecules, changing the ortho/para ratio, as well as causing attenuation or enhancement of the libration frequencies of water molecules, etc. It plays a significant role in determining the efficiency of MWT, resulting in low reproducibility, the presence of deviations between the laboratory and production tests, inconsistency of research results, which is marked in many papers [Lipus, et al., 2013; Chibowski, et al., 2018; Kozic, et al., 2010; Zaidi, et al., 2013; Bali, et al., 2018; Liu, et al., 2015; Toledo, et al., 2008; Seyfi, et al., 2017; Al-Ogaidi, et al., 2017; Szcześ, et al., 2011]. On the basis of presented hypothesis, it was possible to improve the water treatment processes in different ways (Fig. 3).

A systematic approach to studying the MWT effects conducted in this study, taking into account the single results of the previous examples use of MWT in the environmental protection processes [Lipus, et al., 2007; Liu, et al., 2015; Koshoridze, et al., 2014; Kravchuk, et al., 2011; Kozic, et al., 2010; Kilaj, et al., 2018], allowed to build scientifically based provisions for the implementation of the MWT. On the basis of the developing provisions will expand the possibilities for using MWT in the technological processes of water preparation and purification. The application of MWT effects for the intensification of water treatment processes (Fig. 3) is one of the effective methods for reducing the anthropogenic load on the natural water resources. It allows obtaining significant environmental and technical effects:
- ensures the rational use of water resources due to repeated use of water in circulating water supply systems and reduction of the discharges of water with high salt content on account of scale deposition reduction on heat exchange surfaces in the cooling systems of thermal power plants.

- increases the efficiency of thermal installations and ensures the rational use of energy resources on account of preventing the scale deposition on the heat exchange surfaces in heating and hot water systems.

- sharing MWT and ion-exchange filters in the process of removal of dissolved inorganic substances increases the efficiency of the ionexchange filters and reduces the number of regenerations. It allows reducing the discharge of salt water after filter regeneration.

- inactivation of microorganisms under the action of MWT in the processes of disinfection of natural and wastewater. It is used as a pretreatment to reduce the need for reagents in subsequent purification stages.

- increasing the efficiency of the removal of suspended particles from contaminated water during the stages of coagulation, flotation or filtration. It ensures compliance with regulatory requirements, or reduces the need for reagents.

- reduces the needs for reagents and increases the efficiency of purification in the processes of reagent treatment of contaminated water on account of increasing the kinetics of chemical reactions after MWT.

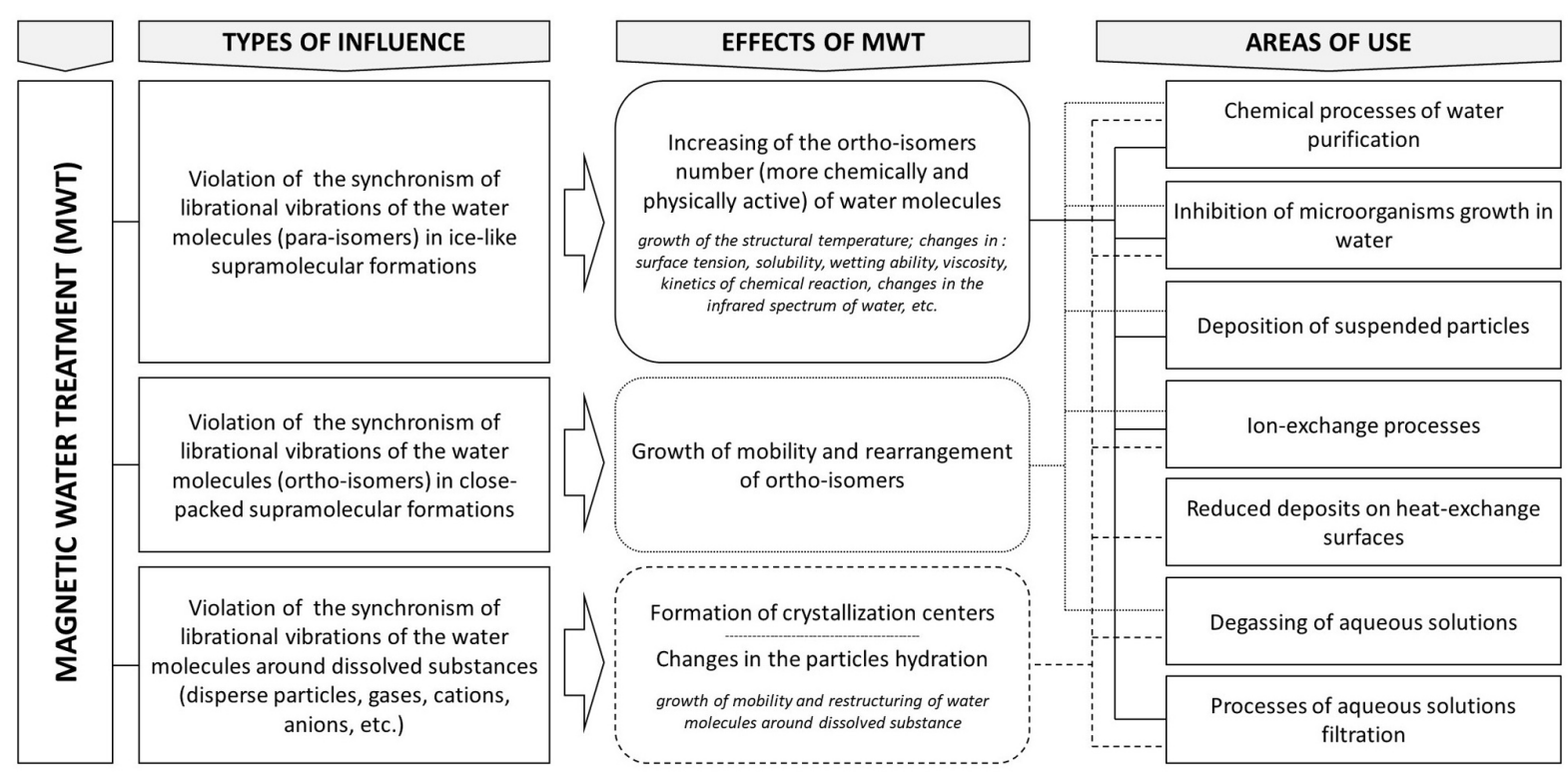

Figure 3. Inhomogeneous magnetic field effects on aqueous solutions and their applications in environmental protection processes 


\section{CONCLUSIONS}

The effects of MWT differ primarily from the underlying phenomenon (reaction kinetics, deposition, scale formation, etc.), the effect of the above-mentioned factors on the MWT efficiency needs further studying. Some perspective ways for further scientific research are following:

- the development of methodological foundations for the implementation of the MWT process,

- experimental confirmation of the influence of different factors on the MWT efficiency.

Mathematical modeling of the process taking into account mineral composition, the presence of dissolved gases, and inorganic concentrations in wastewaters, was conducted.

The physical model of the mechanism of the MWT effects confirms the physicochemical bases of the influence of weak magnetic fields on the properties of aqueous solutions. The model views the aqueous solution as a nonequilibrium dynamic system with the presence of two different states for its molecules. The MWT mechanism underlies the influence of a non-uniform magnetic field on the synchronous libration oscillations of para$\mathrm{H}_{2} \mathrm{O}$ in the ice-like supramolecular formations. The emergence of additional elliptical orbit precession around the $\mathrm{H}$ bonds leads to the destruction of ice-like supramolecular formations, with subsequent conversion of para- $\mathrm{H}_{2} \mathrm{O}$ to ortho- $\mathrm{H}_{2} \mathrm{O}$. Ortho- $\mathrm{H}_{2} \mathrm{O}$ is more physically and chemically active. The increase in their number explains the appearance of the MWT effects.

The proposed physical model allows for the first time to explain the reasons for the decrease in the efficiency of MWT when using high magnitudes of magnetic induction and with increasing the temperature of the treated solutions, to describe the nature of long-term storage of changes in the physical and chemical properties of aqueous solutions after MWT as well as the relatively low reproducibility of the experimental data.

\section{Acknowledgements}

The publication was funded by the Polish National Agency for Academic Exchange under the International Academic Partnerships Programme from the project "Organization of the 9th International Scientific and Technical Conference entitled Environmental Engineering, Photogrammetry, Geoinformatics - Modern Technologies and Development Perspectives".

\section{REFERENCES}

1. Al-Ogaidi A.A.M., Wayayok A., Rowshon M.K., Abdullah, A.F. 2017. The influence of magnetized water on soil water dynamics under drip irrigation systems. Agricultural Water Management, 180 (A), 70-77.

2. Bali M., Mabrouk Y., Abdelli N. 2016. Effects of magnetic treatment on scaling power of hard waters. Separation and Purification Technology, 171, 88-92.

3. Bali, M., Gueddari, M. 2018. The effect of magnetic treatment on the physico-chemical and microbiological characteristics of hard waters. Separation Science and Technology, 53(9), 1405-1411. https:// doi.org/10.1080/01496395.2018.1444640

4. Chibowski, E., Szcześ, A. 2018. Magnetic water treatment - A review of the latest approaches. Chemosphere, Vol. 203, 54-67.

5. Colic, M., Morse, D. 1999. The elusive mechanism of the magnetic 'memory' of water. Colloids and surfaces A: Physicochem, Eng. Aspects 154, 167-174.

6. Hama, T., Kouchi,A., Watanabe, N.. 2016. Statistical ortho-to-para ratio of water desorbed from ice at 10 kelvin. Science, 351 (6268), 65-67. DOI: 10.1126/ science.aad4026

7. Horke, D.A., Chang, Y.-P., Długołęcki, K., Küpper, J. 2014. Separating Paraand Ortho Water. Angewandte Chemie International Edition, 53 (44), 11965-11968. DOI:10.1002/anie.201405986

8. Kilaj, A., Gao, H., Rösch, D., Rivero, U., Küpper, J., Willitsch, S. 2018. Observation of different reactivities of para and ortho-water towards trapped diazenylium ions. Nature communications, 9:2096, 1-7. DOI: $10.1038 / \mathrm{s} 41467-018-04483-3$

9. Klassen, V. 1982. Magnetization of Water Systems. Moskow, Nauka, 296 p.

10. Koksharov, S.A., Konstantinov, O.I., Moriganov, A.P., Melnikov, B. . 1990. Vliyanie magnitnogo polya na sostoyanie rastvorov aktivnyh krasiteley [Effect of a magnetic field on the state of active dye solutions]. Journal prikladnoy himii, 63 , (3), 565-571.

11. Koshoridze S.I., Levin Yu.K. 2014. Model of scale deposition with magnetic water treatment. Formerly known as nanomechanics science and technology: an international journal, 5(1), 51-71.

12. Kozic, V., Hamler, A., Ban, I., Lipus, L.C.. 2010. Magnetic water treatment for scale control in heating and alkaline conditions. Desalination and Water Treatment, 22(1-3), 65-71. https://doi.org/10.5004/ dwt.2010.1549

13. Kravchuk T., Reznikov M., Tichonov P., Avidor N., Meir Y., Bekkerman A., Alexandrowicz G.. 2011. A Magnetically Focused Molecular Beam of 
Ortho-Water. Science, 331(6015), 319-321. DOI: 10.1126/science. 1200433

14. Kuyanov-Prozument, K., Choi, M.Y., Vilesov, A.F.. 2010. Spectrum and infrared intensities of $\mathrm{OH}-$ stretching bands of water dimmers. J. of Chem. Phys, 132, p. 014304

15. Lipus L. C., Acko B., Neral B. 2013. Influence of magnetic water treatment on fabrics' characteristics. Journal of Cleaner Production, 52, 374-379.

16. Lipus L. C., Dobersek D. 2007. Influence of magnetic field on the aragonite precipitation. Chemical Engineering Science, 62, 2089-2095.

17. Liu, Z., Liang, Z., Wu, S., Liu, F.. 2015. Treatment of municipal wastewater by a magnetic activated sludge device. Desalination and Water Treatment, 53(4), 909-918. https://doi.org/10.1080/19443994.2013.848416

18. Malafaev, N.T. 2011. Interactions and dynamics of molecules in pure water [in Russian]. VostochnoEvropeyskiy journal peredovyh tehnologiy, 4(8), 48-58.

19. Malafaev, N.T., Pogojih, N.I. 2015. Modeling of rotational vibrations of water molecules [in Russian]. Vostochno-Evropeyskiy journal peredovyh tehnologiy, 2(5), 27-35.

20. Malafaev, N.T., Pogojih, N.I., Ishtvan, E. A. 2013. Features of rotational vibration modes of water molecules in free and bound states [in Russian]. Vostochno-Evropeyskiy journal peredovyh tehnologiy, 5(6), 11-15.

21. Mamrashev A.A.; Maximov L.V.; Nikolaev N.A.; Chapovsky P.L. 2018. Detection of Nuclear Spin Isomers of Water Molecules by Terahertz Time-Domain Spectroscopy. IEEE Transactions on Terahertz Science and Technology, 8(1), 13-18. DOI: 10.1109/TTHZ.2017.2764385

22. Meier, B., Kouřil, K., Bengs, C., Kouřilová, H., Barker, T.J., Elliott, S.J., Alom, S., Whitby, R.J., Levitt, M.H.. 2018. Spin-isomer conversion of water at room temperature, and quantum-rotor-induced nuclear polarization, in the water-endofullerene. Phys. Rev. Lett. 120, 266001 (6 pp). DOI: 10.1103/ PhysRevLett.120.266001

23. Pershin S.M., Grishin M.Ya, Lednev V.N., Garnov S.V., Bukin V.V., Chizhov P.A., Khodasevich I. A., Oshurko V. B. 2018. Quantification of distortion of the water $\mathrm{OH}$-band using picosecond Raman spectroscopy. Laser Physics Letters, 15(3), 035701 (4pp). DOI: 10.1088/1612-202X/aa9321
24. Pershin, S.M. 2012. Quantum differences between the ortho and the para of spin isomers of $\mathrm{H} 2 \mathrm{O}$ as the physical basis of the anomalous properties of water [in Russian]. Nanostructures. Mathematical Physics and Modeling, 7(2), 103-120.

25. Plyatsuk L.D., Roy I.O., Chernysh Y.Y., Kozii I.S., Hurets L.L., Musabekov A.A. 2019. Clarification of the recent scientific approaches in magnetic water treatment. Journal of Engineering Sciences, 6(1), F12-F18. DOI: 10.21272/jes.2019.6(1).f3

26. Rashid, F.L., Hassan, N.M., Jafar, A.M., Hashim, A.. 2013. Increasing Water Evaporation Rate by Magnetic Field. International Science and Investigation Journal, 2(3), 61-68.

27. Roi, I.O. 2014. c sampling in the processes of natural water treatment [in Russian]. Ecologiya i promyshlennost, 3(40), 47-52.

28. Sammer, M., Kamp, C., Paulitsch-Fuchs, A.H., Wexler, A.D., Cees, J., Buisman, N., Fuchs, E.C.. 2016. Strong Gradients in Weak Magnetic Fields Induce DOLLOP Formation in Tap Water. Water, 8(3):79, 1-19. DOI:10.3390/w8030079

29. Seyfi A., Afzalzadeh R., Hajnorouzi A. 2017. Increase in water evaporation rate with increase in static magnetic field perpendicular to water-air interface. Chem. Eng. Process, 120, 195-200.

30. Szcześ A., Chibowski E., Holysz L., Rafalski P. 2011. Effects of static magnetic field on water at kinetic condition. Chemical Engineering and Processing: Process Intensification, 50 (1), 124-127.

31. Toledo, E.J.L., Ramalho, T.C., Magriotis, Z.M.. 2008. Influence of magnetic field on physicalchemical properties of the liquid water: Insights from experimental and theoretical models. Journal of Molecular Structure, 888(1-3), 409-415. DOI:10.1016/j.molstruc.2008.01.010

32. Voloshyn, V.P., Jeligovskaia, E.A., Malenkov, G.G., Naberuhin, J.I. Tytik, D.L. 2001. Hydrogen bond network structures and the dynamics of water molecules in condensed water systems [in Russian]. Rossiyskiy Himicheskiy Journal, Vol. XLV, 3, 31-37.

33. Zaharov, S. D., Mosiagina, I. V. 2011. Cluster structure of water [in Russian]. Moscow, p. 24.

34. Zaidi, N.S., Sohaili, J., Muda, K., Sillanpää, M.. 2013. Magnetic Field Application and its Potential in Water and Wastewater Treatment Systems. Separation \& Purification Reviews, 43(3), 206-240. https://doi.org/10.1080/15422119.2013.794148 